\title{
TARIFICACIÓN DE la Transmisión ElÉCTRICA USANDO Factores GGDF y GLDF: UnA Estimacion DE SUS EFECTOS Distributivos*
}

\author{
Alexander Galetovic \\ RodRIGo PALMA \\ Universidad de Chile
}

\begin{abstract}
Los factores de distribución GGDF y GLDF son un método atractivo para prorratear los peajes que deben pagar los usuarios de sistemas eléctricos de transmisión. Sin embargo, se demuestra que el método no es suficiente para definir un único prorrateo. Los grados de libertad inherentes a la metodología permiten alcanzar prácticamente cualquier prorrateo de los cargos eligiendo adecuadamente las reglas de asignación entre inyecciones y retiros y parámetros asociados.

Haciendo uso del Sistema Interconectado Central Chileno (SIC), se estudió la forma en que varía la distribución de los cargos de transmisión para reglas y parámetros específicos. El resultado principal es que ésta depende en forma crucial de la fracción del uso total atribuida a las inyecciones y los consumos.
\end{abstract}

JEL: L51, L94

Palabras clave: Tarificación Eléctrica, Efectos Distributivos, Percentiles, Prorrata, Umbral de Corte.

\section{INTRODUCCIÓN}

Conceptualmente hablando, la tarificación de las líneas de transmisión eléctrica aún es materia no resuelta, en gran medida, porque no existe claridad sobre cuál es la economía básica de los sistemas de transmisión. Por eso, en el mundo se

* Este trabajo fue financiado parcialmente por AES Gener S.A. Rodrigo Palma también agradece el financiamiento de Fondecyt (proyecto 1020801). Sin embargo, su contenido es de nuestra exclusiva responsabilidad y no compromete de manera alguna a AES Gener S.A. o a Fondecyt. Agradecemos la ayuda de Carlos Aguirre y Cristián Muñoz, de Gener, y de Juan Pérez, de la Universidad de Chile. También estamos muy agradecidos de los comentarios de Andrea Butelmann, Juan Ricardo Insotroza, un árbitro anónimo y los participantes de las Terceras Jornadas de Derecho Eléctrico, y la sesión especial sobre transmisión eléctrica en el Encuentro de la Sociedad de Economía de Chile. 
usa una gran variedad de mecanismos que difieren apreciablemente entre sí. Esta indeterminación se ha reflejado durante la reciente discusión sobre la así llamada "ley corta" que modificará la regulación de la transmisión eléctrica y probablemente explica la sucesión de mecanismos y reglas de asignación propuestas desde que el proyecto de ley se envió al Congreso en mayo de 2002. ${ }^{1}$ Si bien desde el principio ha estado claro que los peajes se repartirán entre quienes deben pagarlos usando los métodos GGDF (por generalized generation distribution factors) y GLDF (por generalized load distribution factors), éstos, se verá, no pueden ser implementados sin especificar una regla de asignación y una serie de parámetros técnicos. El propósito de este trabajo es explorar qué tan sensibles son los pagos de peajes de cada agente a la regla de asignación elegida y a los parámetros específicos necesarios para implementarla cuando son distribuidos usando estos factores.

El punto de referencia de nuestro estudio es el Sistema Interconectado Central (SIC) en el año 2002. Para ese año estimamos el pago efectivo de peajes que hicieron los distintos generadores de acuerdo con sus contratos, y los comparamos con los que hubieran hecho con reglas de asignación alternativas que reparten los peajes usando factores GGDF y GLDF. Estas reglas son, grosso modo, las que se propusieron en sucesivas versiones de la ley corta hasta enero de 2003.

La estimación del pago de peajes que le corresponde a cada agente se hace en tres etapas. Primero, se estiman los flujos de potencia en cada tramo para un conjunto representativo de posibles condiciones de operación. El conjunto de condiciones de operación busca reflejar el "uso" del sistema troncal que hace cada agente, generador o consumidor. Estos flujos varían según el mes del año, la hora del día y la condición hidrológica, quedando determinados por el despacho definido para las distintas centrales. Segundo, para cada central del sistema y cada consumo se determina su participación en el flujo de potencia para cada condición de operación del sistema (o "uso" del sistema de transmisión). Tercero, utilizando la regla de asignación correspondiente y los valores de cada tramo del sistema de transmisión, se determina el pago de peaje que le corresponde a cada agente, ya sea central o carga. El resultado de estos cálculos se compara con los pagos de peajes con los contratos vigentes en 2002 que fueron negociados con la regulación actual.

La principal conclusión del trabajo es que, eligiendo apropiadamente la regla de asignación y ciertos parámetros, los factores GGDF y GLDF permiten lograr casi cualquier asignación de peajes entre los agentes del sistema. La indeterminación indica que, seguramente, estos métodos permiten seleccionar parámetros que inducen pagos de peajes económicamente sensatos y dan señales económicas apropiadas. Pero el marco conceptual que determina lo económicamente apropiado es anterior a los métodos GGDF o GLDF y debe definirse antes de fijar los parámetros específicos. En otras palabras, estos métodos pueden ser

\footnotetext{
${ }^{1}$ La versión final de este trabajo se entregó en noviembre de 2003 y por razones obvias no incluye las discusiones posteriores. Para un análisis de la regulación de transmisión en la ley corta, véase Galetóvic y Muñoz (2004).
} 
apropiados para prorratear los peajes de una línea determinada, pero sólo si antes se ha decidido quién debiera pagarlos de acuerdo con la lógica económica del problema. Por el contrario, son inadecuados para seleccionar, entre todos los agentes que participan del sistema, quién debe pagar una línea determinada.

El proyecto de ley corta que actualmente se discute en el Congreso (noviembre de 2003) se hace cargo en buena medida de estos problemas, y restringe la posterior discreción del regulador fijando reglas de asignación explícitas que indican cómo se debe determinar la participación de inyecciones y retiros en cada tramo del sistema de transmisión. ${ }^{2}$ Sin embargo, para evitar la discrecionalidad, es importante que las reglas específicas que deban precisarse en reglamentos y resoluciones sean discutidas y fundamentadas transparentemente.

Luego de esta introducción, en la sección 2 discutimos brevemente un par de conceptos básicos necesarios para entender la discusión sobre quién paga los peajes. En la sección 3 explicamos con mayor detalle la metodología que usamos para hacer los cálculos. En la sección 4 describimos en detalle los supuestos y las fuentes de datos que utilizamos para hacer los cálculos. La sección 5 presenta los resultados, y la 6 las conclusiones. Un apéndice explica cómo dedujimos el pago actual de peajes de cada grupo de generadores.

\section{Conceptos: ¿Quién Paga una Línea de Transmisión?}

En este trabajo queremos estudiar la distribución de peajes bajo reglas de asignación alternativas. Pero para hacerlo es necesario preguntarse antes: ¿quién paga cada línea? Para responder a esta pregunta es necesario definir un par de conceptos básicos: regla de asignación, uso de una línea de transmisión, sujeto de pago y cargo directo. ${ }^{3}$

Como se puede apreciar en el Cuadro 1, una regla de asignación de peajes indica dos cosas. Primero, el sujeto de pago, vale decir, quién es el encargado de "hacer el cheque" que recibe el transmisor. En segundo lugar, también indica quién usa cada tramo del sistema de transmisión. Grosso modo, el uso se le atribuye a las centrales que inyectan, a los consumos que retiran, o bien, a una combinación de ambos.

El Cuadro 1 muestra una regla cualquiera y arbitraria de asignación del peaje de una línea hipotética. Una manera de mirar la regla de asignación es sumando las filas de la matriz, las que determinan el pago por uso. Se aprecia que el 52\% del uso de esa línea se le atribuye a las inyecciones de centrales y el $48 \%$ restante a los retiros.

Al mismo tiempo, la regla de asignación se puede mirar sumando las columnas de la matriz, las que indican cuánto paga cada sujeto. Haciéndolo, se obtiene que el transmisor cobrará el $40 \%$ de los peajes a generadores y el $60 \%$ restante a consumidores. ¿Cómo se descomponen estas sumas?

\footnotetext{
${ }^{2}$ Un análisis de este proyecto se encuentra en Galetovic y Muñoz (2004).
} 
La celda $(1,1)$ de la matriz indica que el $21 \%$ del peaje de esta línea se le cargará a las inyecciones que la usan y será pagado por los generadores (los sujetos de pago). Con algún método se determina el uso de cada generador y éste, a su vez, determina la prorrata de cada uno. El $21 \%$ se reparte entre los generadores de acuerdo a la prorrata de cada uno.

\begin{tabular}{lcc}
\multicolumn{3}{c}{ CUADRO 1 } \\
\multicolumn{3}{c}{ REGLAS DE ASIGNACION } \\
\hline & \multicolumn{2}{c}{ Sujeto de pago } \\
& Generadores & Usuarios \\
\hline Inyecciones & $21 \%$ & $31 \%$ \\
Retiros & $19 \%$ & $29 \%$ \\
\hline
\end{tabular}

La celda $(1,2)$ de la matriz indica que la responsabilidad de pagar el $31 \%$ de la línea será de los consumidores (el sujeto de pago), pero la prorrata de cada uno se determinará según el uso que hagan los generadores que lo abastecen. El uso de cada generador es idéntico al de la celda $(1,1)$, pero esta vez la prorrata respectiva se reparte entre los consumidores con quienes tiene contrato cada generador.

La celda $(2,1)$ indica que el $19 \%$ de la línea se le cobrará a los generadores por el uso de la línea que hagan sus clientes. Con algún método se determina el uso de cada consumidor y el $19 \%$ se reparte de acuerdo con la prorrata de cada uno. La prorrata de cada consumidor se le asigna al generador con quien tenga contrato.

Por último, la celda $(2,2)$ indica que el $29 \%$ del peaje de esta línea se le cargará a los consumidores que la usan y será pagado por ellos (los sujetos de pago). El uso de cada consumidor es idéntico al de la celda $(2,1)$, pero esta vez cada consumidor es responsable de pagarle al transmisor su prorrata.

¿Qué se entiende por “uso"? Cuando se trata, por ejemplo, de una carretera el uso es fácil de determinar, porque es identificable que un automóvil va desde Santiago hacia Valparaíso. Sin embargo, cuando se trata de sistemas eléctricos, el uso es difícil de definir, porque cuando una central inyecta un $\mathrm{KW}$ adicional o un consumo lo retira, los flujos se ajustan y varían en todo el sistema obedeciendo a las leyes de Kirchhoff. De esta forma, no se pueden identificar físicamente los electrones inyectados por una central. Por esta razón fundamental los métodos que existen para determinar el uso de una línea son descomposiciones contables de los flujos (contables en el sentido que obedecen ciertas reglas básicas de consistencia) que, sin embargo, se deducen de propiedades eléctricas. Para determinar el uso que hace de cada línea cada central generadora, usaremos el método de los GGDF. Similarmente, para determinar el uso que hace cada consumidor de cada línea se empleará el método de los GLDF.

\footnotetext{
${ }^{3}$ Una discusión más detallada se encuentra en Galetovic y Palma (2004).
} 
¿Por qué distinguir entre el sujeto de pago y la regla para determinar el uso? La razón es que la incidencia de una regla de asignación -vale decir, quién paga finalmente una línea-y, por lo tanto, sus efectos económicos dependen del criterio que se use para determinar quién "usa" determinada línea, y no de quiénes sean los sujetos de pago. En efecto, mientras la regulación no sea expropiatoria fijando precios por debajo de los costos, en el largo plazo los usuarios terminarán pagando los peajes de transmisión casi con independencia de quién sea el sujeto de pago. ${ }^{4}$ Sin embargo, la regla para determinar el uso afectará el costo relativo de localizar centrales y, en menor medida, consumos, en distintos puntos del sistema. Por ejemplo, si se pasa de un criterio que le asigna el 100\% de un tramo dado a las inyecciones a uno en que las inyecciones pagan sólo el 50\% y el resto los retiros, inmediatamente se hace más barato localizar una central en el extremo de ese tramo, independientemente de si los sujetos de pago del 50\% restante son empresas generadores o consumidores. A continuación argumentamos que, en el largo plazo, la incidencia sólo depende de la regla de asignación, no de quién sea el sujeto de pago.

Considérese primero la celda $(2,2)$ : uso asignado a retiros que deben pagarle directamente al transmisor. Claramente, el pago asignado a los retiros es irrelevante desde el punto de vista de la competencia entre generadores: el pago por transmisión lo hace el cliente. Por lo mismo, a pesar de que en la celda $(2,1)$ el sujeto de pago es el generador, la competencia debiera llevar a que el peaje fuera irrelevante. En efecto, supóngase que un grupo de ellos compite por un cliente cuyo peaje asignado es $\tau$. El peaje $\tau$ formará parte de los costos de quien gane la licitación y, por lo tanto, debiera ser traspasado al precio que pagará el cliente.

Por otro lado, es razonable suponer que el pago asignado a las inyecciones no se traspasará directamente a los precios que pagan los consumidores, porque estos pagos difieren entre centrales según dónde se ubiquen. Esto es así, porque, por las características del despacho, el costo de energía y potencia para servir un contrato en un nudo determinado es el mismo para todos los generadores, independientemente de los peajes que paguen sus centrales. En competencia, por lo tanto, el cargo de transmisión deberá ser pagado por la central.

En este trabajo no estudiaremos estos efectos sobre la asignación de recursos. Nos limitaremos a estimar los peajes que hubieran pagado distintos agentes -empresas generadoras y consumos-en 2001 y 2002 con distintas reglas de asignación, y las compararemos con las que pagaron con la regulación actual. Por supuesto, esto nos obligará a suponer lo que no conocemos, en particular, la fracción de los peajes por cuenta de retiros que las empresas generadoras podrán traspasarle a sus clientes con contratos vigentes.

\footnotetext{
${ }^{4}$ Por supuesto, en la transición desde un mecanismo de reglas de tarificación a otro seguramente ocurrirán redistribuciones de riqueza mientras vencen los contratos ya firmados. Por eso, el traspaso a consumidores probablemente será gradual.
} 


\section{Metodología}

En esta sección describimos la metodología que usaremos para obtener las estimaciones de pago de peajes. Esta estimación se hace en tres etapas: (i) determinación de las condiciones de operación y los flujos por cada línea; (ii) asignación entre los agentes conectados al sistema de los flujos en cada línea para cada condición de operación y cálculo de las prorratas respectivas; (iii) cálculo del pago que le corresponde a cada agente. Describimos cada etapa a continuación, distinguiendo los diferentes esquemas que ha ido proponiendo la CNE.

\subsection{Las Condiciones de Operación}

Conceptos básicos de operación. Los sistemas eléctricos chilenos se operan centralizadamente para minimizar el costo esperado de abastecimiento y falla. ${ }^{5}$ El CDEC despacha las centrales en "orden de mérito", vale decir, las ordena de menor a mayor costo de operación y las va haciendo entrar sucesivamente hasta cubrir la cantidad demandada en cada momento. En el caso del SIC, primero entran en funcionamiento las centrales hidráulicas de pasada. Su costo variable de operación es prácticamente nulo: no tiene costo alternativo, porque no pueden embalsar agua. Si la cantidad producida por centrales de pasada no es suficiente, entran en funcionamiento centrales térmicas en orden creciente de costos de operación y de embalse, de acuerdo con el costo de oportunidad del agua embalsada en ese momento.

Para minimizar el costo social de abastecimiento, la regulación estipula que las órdenes del CDEC son obligatorias e independientes de los contratos de comercialización de cada empresa. Por ello, es frecuente que existan transferencias entre generadores para que puedan cumplir con sus compromisos comerciales. Estas se valoran al costo marginal instantáneo del sistema o preciospot calculado cada hora. Esta separación entre despacho y contratos permite que el sistema minimice el costo total de producción. Lo que es más importante en este trabajo, implica que el flujo de potencia por cada línea de transmisión es determinado únicamente por la hidrología, los consumos en los distintos nodos del sistema y el uso de agua del lago Laja, y no por los contratos entre generadores y usuarios. ${ }^{6}$

Esto se puede apreciar en el Gráfico 1. Cuánto se genera con centrales térmicas en cada momento depende del nivel de la demanda, de la disponibilidad de generación hidráulica de pasada y de la cantidad generada con agua embalsada. Así, mientras más generan las centrales del Laja, o más abundante es la hidrología, menos se genera con centrales térmicas. En particular, en este trabajo supondremos que en cada mes hay tres niveles de demanda representado cada

\footnotetext{
${ }^{5}$ Una descripción detallada del modelo Omsic utilizado ampliamente en el sector eléctrico se encuentra en Galetovic, Olmedo y Soto (2002).

${ }^{6}$ Este diseño de mercado se conoce internacionalmente como "pool obligatorio con costos auditados". Este modelo no se usa en países como Alemania o los Estados Unidos, donde se enfatizan los contratos bilaterales físicos entre empresas generadoras y consumidores.
} 
uno por un bloque de demanda (alto, medio y bajo); al mismo tiempo, en cada mes se supondrá que son posibles 40 hidrologías; finalmente, cada mes es hidrológicamente distinto. Así, tenemos $3 \times 12 \times 40=1.440$ condiciones de operación, las que denotaremos con la letra $t$.

\section{GRAFICO 1 \\ DESPACHO POR ORDEN DE MERITO}

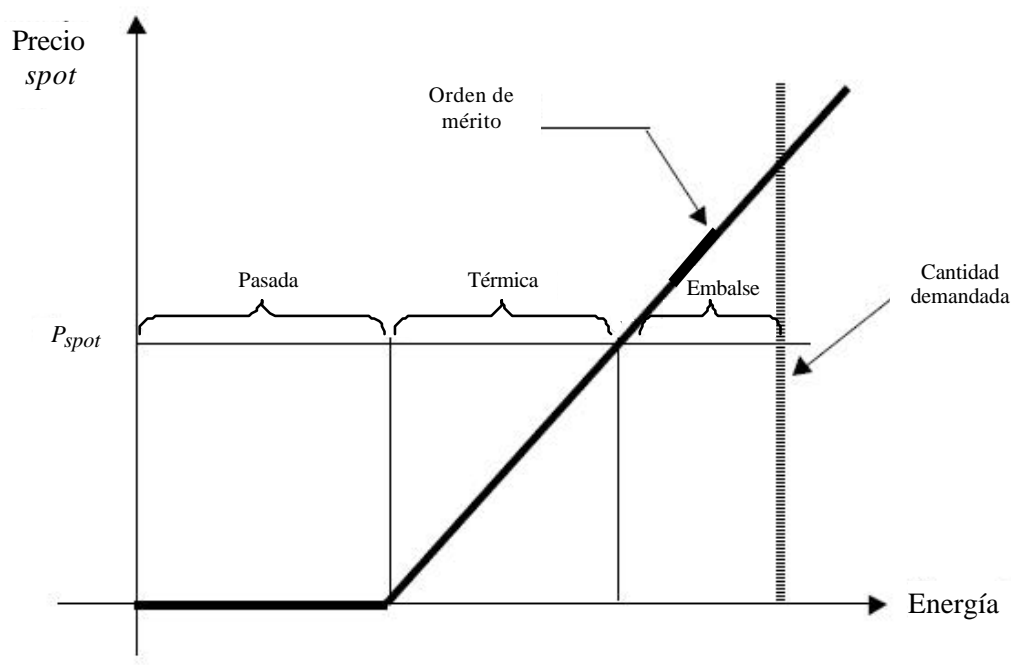

¿En qué consiste exactamente una “condición de operación”? Si $G_{g, b}(t)$ es la potencia generada por la central $g$ que inyecta en la barra $b$ en la condición de operación $t$, podemos denotar por $G(t)$ al vector-columna que incluye lo generado por cada una de las $N$ centrales conectadas al sistema en la condición de operación $t$. Por otro lado, $\operatorname{si}_{c, b}(t)$ es el retiro del consumo $c$ ubicado en la barra $b$, podemos denotar por $C(t)$ al vector-columna que incluye lo retirado por cada una de las $M$ cargas conectadas al sistema en la condición de operación $t$. Así, cada condición de operación consiste en un vector-columna $G(t)$ que indica las inyecciones de cada generador y un vector-columna $C(t)$ que indica los retiros de cada consumo o carga.

La ley obliga a usar en todo momento la cantidad tal que se sirva la demanda al mínimo costo social esperado de abastecimiento y falla. Esta cantidad óptima la calcula el CDEC usando el modelo de programación dinámica estocástica Omsic (acrónimo de "operación mensual del SIC"), que optimiza el uso del agua del lago Laja, en conjunto con los modelos de embalse de Colbún, Pehuenche y Rapel. Nosotros utilizaremos el modelo Omsic para simular los despachos y la operación del sistema y obtener las 1.440 condiciones de operación. ${ }^{7}$

${ }^{7}$ El lago Laja tiene capacidad para almacenar el equivalente a 7.000 GWh de energía (aunque la capacidad de generación anual es alrededor de $2.500 \mathrm{GWh}$ a través de las centrales El Toro, Antuco y Abanico) y se puede usar para guardar agua por varios años. Para más detalles véanse Galetovic, Olmedo y Soto (2002) y Olmedo (2001). 
Flujos por cada línea. El modelo Omsic es uninodal, vale decir, supone que todo el despacho de centrales ocurre en un solo nodo del sistema y, por lo tanto, las pérdidas no se obtienen de la solución del modelo en función de la ubicación de los despachos y las inyecciones. Sin embargo, la estimación de demanda del sistema que se usa para determinar el despacho incluye un factor de pérdidas; es decir, las pérdidas afectan el monto generado. Para incluir las pérdidas ajustamos el vectorcolumna de retiros $C(t)$ prorrateando la generación total del sistema entre cada uno de los consumos. El prorrateo es distinto para cada bloque de demanda y cada mes (vale decir, se utilizan $3 \times 12=36$ esquemas de prorrateo) e independiente de las inyecciones.

En conjunto, las inyecciones y los retiros resumidos por el par $\{C(t), G(t)\}$ determinan el flujo de potencia en la línea que une los puntos $l$ y $k$ en la condición de operación $t$, que denotaremos por $P_{l \rightarrow k}(t)$. Denotamos por $P(t)$ el vectorcolumna de dimensión $L$ que incluye los flujos en cada una de las $L$ líneas del sistema bajo la condición de operación $t$.

Dadas las cantidades generadas y consumidas, y su localización eléctrica para cada condición de operación, utilizaremos el modelo "flujo de potencia en continua o flujo lineal DC" para obtener los 1.440 vectores $P(t)$. Este modelo es lineal y permite aproximar la operación de un sistema eléctrico de potencia que normalmente se representaría por un sistema de ecuaciones no lineales. Una aproximación de este tipo es razonablemente precisa en sistemas de alta tensión como el estudiado. Adicionalmente, el modelo de flujo supone que no hay pérdidas. Este supuesto es consistente, porque los consumos obtenidos del modelo Omsic ya incluyen, en forma aproximada, las pérdidas óhmicas del sistema.

En definitiva se dispone de un sistema lineal que relaciona las inyecciones netas de potencia (generación menos consumo) en cada uno de los nudos del sistema con los flujos de potencia en cada una de las líneas de transmisión. Las simulaciones se hacen con un modelo simplificado del SIC de 463 barras, 438 líneas, 158 transformadores, 58 generadores y 258 cargas.

\subsection{El "uso" de las Líneas: los GGDF y los GLDF}

Una vez determinados los flujos de cada tramo en cada condición de operación (los 1.440 vectores $P(t)$ ), obtenemos el "uso" que hace cada agente del sistema de transmisión bajo cada condición de operación con los factores GGDF y GLDF. ${ }^{8}$

Centrales e inyecciones. El "uso" de cada línea que hace una central en cada condición de operación se determina con los factores GGDF. A continuación describimos cómo obtendremos la prorrata de cada central en cada tramo de interés en función de los factores GGDF.

\footnotetext{
${ }^{8}$ Ver Ng (1981) y Rudnick et al. (1995). En el apéndice B de Galetovic y Palma (2003) presentamos una breve introducción a los factores GGDF y deducimos algunas de sus propiedades.
} 
Se puede demostrar que el flujo de potencia en la línea que une los puntos $1 \mathrm{y} \mathrm{k}$ se puede descomponer de la siguiente forma:

$$
P_{l \rightarrow k}(t)=\sum_{g=1}^{N}\left[D_{l \rightarrow k}^{b} \times G_{g, b}(t)\right]
$$

donde $D_{l \rightarrow k}^{b}$ es el factor GGDF para la línea $l \rightarrow k$, el mismo para cada uno de los generadores que inyectan en la barra $b$. Si $D$ es la matriz de orden $L \times N$ de factores GGDF, entonces se sigue de (3.1) que

$$
P(t)=D \times G(t)
$$

Los factores GGDF tienen tres propiedades que conviene describir. En primer lugar, la matriz $D$ es independiente de la barra de referencia del sistema, pero depende de la condición de operación $t$. Sin embargo, se puede demostrar que cuando se utiliza una aproximación lineal DC del sistema de transmisión y se supone que no hay pérdidas, los factores GGDF sólo dependen de la distribución de los consumos en el sistema y no del despacho de las centrales generadoras. En el trabajo esto es válido para las 40 hidrologías de cada bloque de simulación, ya que la distribución de cargas o consumos es única y no depende de la hidrología ni de los despachos. Para simplificar la notación, en las fórmulas de prorrateo discutidas a continuación se supondrá que los factores GGDF no dependen de la condición de operación estudiada.

En segundo lugar, los factores GGDF son únicos, vale decir, existe una sola matriz $D$ tal que $P(t)=D \cdot G(t)$.

En tercer lugar, el factor GGDF de una central puede ser negativo en algunas líneas, por ejemplo, cuando una central inyecta en un nudo que es importador neto.

Considerando las propiedades anteriores, es posible definir la prorrata que le corresponde a la central g en la línea $l \rightarrow k$ bajo la condición de operación $t$. Esta prorrata la denotamos por $\lambda_{l \rightarrow k}^{g}(t)$ y se define como

$$
\lambda_{l \rightarrow k}^{g}(t)=\frac{D_{l \rightarrow k}^{b} \times G_{g, b}(t)}{D_{l \rightarrow k} \cdot G(t)}=\frac{D_{l \rightarrow k}^{b} \times G_{g, b}(t)}{P_{l \rightarrow k}(t)}
$$

por definición, las prorratas suman uno. Ahora bien, la prorrata de cada una de las $N$ centrales en el tramo $l \rightarrow k$ en la condición de operación $t$ se puede agrupar en el vector-fila de dimensión $N, \Lambda_{l \rightarrow k}(t)$. A su vez, estos vectores se agrupan en la matriz $\Lambda(t)$ de orden $L \times N$. Por definición, cada fila de la matriz suma uno y la fila $l \rightarrow k$ corresponde a las prorratas de cada central en la línea $l \rightarrow k$. Al mismo tiempo, la columna $g$ de esta matriz indica las prorratas de la central $g$ en cada una de las líneas del sistema.

A modo de ejemplo, si un sistema se compone de dos centrales y tres líneas, 


$$
\Lambda(t)=\left[\begin{array}{ll}
\lambda_{1}^{1}(t) & \lambda_{1}^{2}(t) \\
\lambda_{2}^{1}(t) & \lambda_{2}^{2}(t) \\
\lambda_{3}^{1}(t) & \lambda_{3}^{2}(t)
\end{array}\right]
$$

Así, la fila 2 entrega las prorratas de cada una de las centrales en la línea 2, mientras que la columna 2, la prorrata de la central 2 en cada una de las tres líneas.

Es importante notar que, sin perjuicio de ser únicos, los factores GGDF (y también los factores GLDF) son una descomposición "contable" y en alguna medida arbitraria de los flujos de un sistema eléctrico. Esta descomposición no se deduce de principios económicos, sino eléctricos. Por lo tanto, el marco conceptual que determina lo que es "económicamente apropiado" es anterior a los métodos GGDF o GLDF. Por eso, estos métodos pueden ser apropiados para prorratear los peajes de una línea determinada, pero sólo si antes se ha decidido quién debiera pagarlos de acuerdo con la lógica económica del problema.

Cargas y retiros. El "uso" de cada línea que hace una carga en cada condición de operación se determinará con los factores GLDF, los que se obtienen de manera análoga a los factores GGDF. Así,

$$
P_{l \rightarrow k}(t)=\sum_{c=1}^{M}\left[E_{l \rightarrow k}^{b} \times C_{g, b}(t)\right]
$$

donde $E_{l \rightarrow k}^{b}$ es el factor GLDF para la línea $l \rightarrow k$, el mismo para cada carga $C_{g, b}$ que retira en la barra $b$. De manera similar, la prorrata de la carga c en la línea se define como

$$
\phi_{\ell \rightarrow k}^{c}(t) \equiv \frac{E_{\ell \rightarrow k}^{b} \times C_{c, b}(t)}{E_{\ell \rightarrow k}^{b} C(t)}=\frac{E_{\ell \rightarrow k}^{b} \times C_{c, b}(t)}{P_{\ell \rightarrow k}(t)} .
$$

Por lo tanto, la matriz $\Phi(t)$, de orden $L \times M$ y análoga a $\Lambda(t)$, resume la prorrata de cada carga en cada línea: la fila $l \rightarrow k$ corresponde a las prorratas de cada carga en la línea $l \rightarrow k$; y la columna c entrega las prorratas de la carga en cada línea.

\subsection{El pago que le corresponde a cada agente}

El resultado de las primeras dos etapas es un conjunto de 1.440 tuplas $\{\Lambda(t), \Phi(t), P(t), G(t), C(t)\}$ y vectores. Para determinar el pago anual de peajes que le corresponde a cada agente se necesita conocer, además de los peajes de cada línea, la regla de asignación. Una regla de asignación puede ser simple o compleja, pero generalmente especifica tres cosas:

- Una regla tal que, para cada línea $l \rightarrow k$, elige entre las 1.440 condiciones de operación aquellas que se considerarán para calcular el pago de peajes de 
cada agente. Por ejemplo, en este trabajo utilizaremos, entre otras, la regla del percentil superior. Consiste en: (i) ordenar los módulos de los 1.440 flujos de potencia en la línea $l \rightarrow k,\left|P_{l \rightarrow k}(t)\right|$, de mayor a menor; (ii) seleccionar aquellos que estén en el percentil $p$ o superior. Así, si $P=90$, la regla selecciona los 144 mayores flujos de potencia. Denotaremos por $\tau_{l \rightarrow k}$ al conjunto de condiciones de operación a las que se ocuparán para calcular el pago de peajes por la línea $l \rightarrow k$ de cada agente dada la regla. $\tau$ es el vector de dimension $L$ que resume las condiciones de operación para cada una de las $L$ líneas que serán utilizados para calcular los peajes.

- Los ajustes que se le harán a las prorratas. Un ajuste típico, que también haremos en este trabajo, consiste en excluir las prorratas negativas que se deben a los factores negativos por causa de los contraflujos. Así, se sustituyen por ceros los elementos negativos de las matrices $\Lambda(t)$ y $\Phi(t)$ y se escalan las restantes prorratas para que cada fila sume uno. Denotaremos estas matrices ajustadas por $\Lambda^{+}(t) y \Phi^{+}(t)$.

También se podrían excluir de la prorrata las centrales (o, según sea el caso, cargas) cuya participación sea menor que un determinado umbral de corte. Por ejemplo, si el umbral de corte es $10 \%$, en cada tramo se excluyen todas aquellas centrales cuya prorrata sin ajustar sea menor que $10 \%$. Así, de manera similar al caso anterior, se sustituyen por ceros los elementos de las matrices $\Lambda(t)$ y $\Phi(t)$ que sean menores que $10 \%$ y se escalan las restantes prorratas para que cada fila sume uno. En este trabajo, sin embargo, siempre supondremos que el umbral de corte es $0 \%$.

- $\quad$ Para cada línea, la fracción del peaje que le será asignada a las inyecciones de centrales (la denotaremos por $\eta_{l \rightarrow k}$ ) y la fracción que le será asignada a los retiros de las cargas $\left(1-\eta_{l \rightarrow k}\right)$. Por ejemplo, en el proyecto de ley corta originalmente enviado al Congreso, $\eta_{l \rightarrow k}=0,5$ para todas las líneas. Por contraste, en la propuesta de la CNE de enero de $2003 \eta_{l \rightarrow k}$ resulta de un cálculo complejo y es distinto para cada tramo. En adelante, denotaremos por $\eta$ al vector de dimensión $L$ que reparte el pago de peajes entre centrales y cargas.

Con el vector y las matrices ajustadas se calculan las prorratas promedio de cada línea, a saber los vectores

$$
\begin{gathered}
\Lambda_{\ell \rightarrow k}^{+}=\frac{\sum_{\tau_{\ell \rightarrow k} \Lambda_{\ell \rightarrow k}^{+}(t)}}{\left|\tau_{\ell \rightarrow k}\right|} \\
\Phi_{\ell \rightarrow k}^{+}=\frac{\sum_{\tau_{\ell \rightarrow k}} \Phi_{\ell \rightarrow k}^{+}(t)}{\left|\tau_{\ell \rightarrow k}\right|}
\end{gathered}
$$

donde $\left|\tau_{l \rightarrow k}\right|$ es la cardinalidad del conjunto $\tau_{l \rightarrow k}$. 
Finalmente, si $\pi_{l \rightarrow k}$ es el peaje total que le corresponde recibir al transmisor por la línea $l \rightarrow k$, el vector

$$
\eta_{l \rightarrow k} \times \pi_{l \rightarrow k} \times \Lambda_{l \rightarrow k}^{+}
$$

entrega el peaje que le corresponde a cada central y

$$
\left(1-\eta_{l \rightarrow k}\right) \times \pi_{l \rightarrow k} \times \Phi_{l \rightarrow k}^{+}
$$

el peaje que le corresponde a cada carga. A partir de las expresiones (8) y (9) se puede obtener trivialmente el pago total de peajes atribuible a cada central y cada carga, y computar el agregado por generador. En eso consisten los resultados que reportamos en la sección 5 .

\section{Supuest os y Fuentes de Datos}

\subsection{El Sistema Troncal}

Las instalaciones incluidas en el sistema troncal. Las instalaciones incluidas en el sistema troncal son aquellas líneas de $220 \mathrm{KV}$ o más entre el nudo Puerto Montt por el sur y el nudo Diego de Almagro por el norte, según lo presentado por la CNE en enero de 2003 (véase CNE [2002]). El detalle de las líneas incluidas en el cálculo está en el Cuadro A.2 del apéndice.

La valorización de las instalaciones del sistema troncal. Para valorizar el sistema troncal se utilizaron los valores nuevos de reemplazo utilizados enInforme de Peajes del CDEC-SIC de enero de 2003 (en adelante CDEC-SIC (2003)). Este informe valora las instalaciones de transmisión instaladas en (2002). Para prorratear se han considerado las anualidades de cada tramo del sistema troncal y sus costos anuales de operación y mantenimiento, descontando una estimación del ingreso tarifario para cada tramo, cuya fuente también es el informe de peajes. Esta estimación se basa en el informe de peajes realizado del CDEC-SIC de diciembre de 2001. Luego de los ajustes, se concluye que el valor total a prorratear es US\$78,95 millones.

\subsection{Las Condiciones de Operación}

Para definir las 1.440 condiciones representativas de operación del sistema en 2002 se usó una versión uninodal del modelo Omsic. La base de datos de entrada al modelo corresponde a la utilizada por la CNE en la fijación de precios de octubre de 2001. De esta forma, los resultados son consistentes con la información disponible y validada a fines de 2001.

El horizonte de planeamiento. La versión del modelo Omsic que usamos modela la variación mensual de la demanda a través de una curva de duración de carga de tres bloques, para un horizonte de siete años. Al final de los siete años se 
empalma con el modelo GOL mediante la curva de valor estratégico a fines del horizonte del estudio. Este procedimiento corresponde a la práctica del CDEC cuando elabora el programa de la operación del sistema. La optimización del modelo GOL se hizo con un horizonte de planeamiento de 10 años (véase CNE, 2001).

La proyección de demanda para el horizonte de planeamiento. La proyección de demanda corresponde a la que hizo la CNE cuando fijó el precio de nudo en octubre de 2001. Esta proyección de consumo supone una tasa media de crecimiento de $7,5 \%$ anual y un factor de pérdidas de $4 \%$.

Los bloques de carga. La demanda total proyectada de energía de cada mes se ha repartido en tres bloques. En cada uno de ellos se supone que la demanda por potencia es pareja, pero difiere entre bloques. Las diferencias de potencia demandada entre bloques aproximan las curvas de carga observadas empíricamente. La demanda de cada bloque es representada como un porcentaje de la demanda del bloque de punta y su duración se expresa en horas. Según el mes que se trate, la duración de los bloques varía entre 240 y 248 horas.

Las hidrologías. Para modelar la aleatoriedad de la hidrología, utilizamos las estadísticas de 40 años hidrológicos, de 1959-60 a 1998-99, elaboradas por el CDEC. ${ }^{9}$ La optimización del modelo Omsic, que es necesaria para obtener la operación del embalse del lago Laja bajo cada condición de operación, se hace utilizando toda la variabilidad hidrológica con etapas de deshielo determinísticas (véase Galetovic, Olmedo y Soto, 2002) para una explicación más detallada de la optimización.

Para operar el sistema y obtener nuestras condiciones de operación tomaremos las $40 \times 12$ condiciones hidrológicas mensuales materializadas en los 40 años hidrológicos entre 1959-60 y 1998-99. Cada mes se divide en tres bloques de demanda, por lo que cada mes aporta tres condiciones de operación. Para la etapa de simulación (véase Galetovic, Olmedo y Soto, 2002) se han utilizado las series históricas. Para el primer año, las cuarenta simulaciones escogidas como resultado corresponden a las 40 secuencias históricas.

La energía que aportan las centrales de pasada y de embalse en cada una de la 40 hidrologías. Los caudales de los ríos se transforman a energía y se obtienen matrices de energía generable. Estas matrices son de orden 40×12 (40 años hidrológicos, cada uno de 12 meses). Por ejemplo, la entrada (1971-72, agosto) de la matriz es la cantidad de energía que hubieran generado las centrales de pasada durante el mes de agosto del año hidrológico 1971-72.

La energía que aportarán las centrales que utilizan agua del resto de los embalses del SIC (Colbún, Cipreses, Canutillar y Rapel) también se resume en matrices de energía generable. Para obtener las condiciones de operación hemos trabajado con matrices mensuales de largo plazo calculadas por el CDEC-SIC.

${ }^{9}$ El año hidrológico comienza en abril y termina en marzo del año siguiente. Así, el año hidrológico 1959-60 comenzó en abril de 1959 y terminó en marzo de 1960. 
Grosso modo, esto equivale a suponer que la generación de cada central durante el mes es igual a la energía generable con el flujo de agua que entra al embalse en el mes para cada hidrología, sin que se genere nada del stock del agua en el embalse entre inicios y fines del año hidrológico.

A diferencia de las centrales de pasada, el agua de los embalses se puede poner en la punta -es decir, su energía se puede trasladar desde horas en que el costo marginal es bajo a horas de punta en que el costo marginal es alto-. Para representare esto, a cada uno se le asignó una prioridad de puesta en punta, como forma de distribuir la energía de la matriz de energía generable a los bloques de mayor demanda según la capacidad de regulación de cada embalse.

La energía en el Laja. La cota inicial del lago Laja es la de principios de octubre de 2001, 1.343,20 m.s.n.m. Esta cota fue utilizada por la CNE cuando fijó los precios de nudo.

La disponibilidad de centrales térmicas. Las centrales térmicas pueden fallar intempestivamente. Para incorporar este hecho ajustamos la capacidad de cada central multiplicándola por (1-tasa de falla). Nótese que en la práctica la central está disponible o no lo está, por lo que claramente se trata de una aproximación imperfecta. La fuente de las tasas de falla es el CDEC-SIC.

La entrada de centrales. La entrada proyectada de centrales afecta la operación del sistema directamente por los aportes de energía que ellas entregan; pero también indirectamente, porque afectan la operación óptima del embalse del lago Laja. Por ejemplo, el anuncio de una nueva central reduce el valor de guardar agua, y lleva a que hoy se genere más con agua embalsada.

El plan de entrada de centrales (o plan de obras) es el que usó la CNE cuando fijó el precio de nudo en octubre de 2001.

Las salidas de centrales por mantenimiento programado. Las centrales deben retirarse del servicio periódicamente para realizar mantenimiento. La información utilizada corresponde a los mantenimientos típicos utilizados por el CDECSIC: 15 días al año las centrales a gas y 20 días al año las carboneras.

El precio de los combustibles. El precio de los combustibles se supone constante durante los diez años optimizados e iguales a los que aparecen en la fijación de precios de nudo de octubre de 2001.

\subsection{El Pago de Peajes}

Peajes con contratos actuales. El monto de peajes que cobró Transelec ${ }^{10}$ en 2001 se obtuvo de su memoria anual (Transelec (2001)). El resto de los cálculos y supuestos está en el apéndice.

10 Transelec es el principal transmisor en alto voltaje en el Sistema Interconectado Central chileno, dueño de la mayoría de las líneas. 
Peajes por cuenta de retiro. Cuando el pago de peajes se determina según retiros, en la mayoría de los casos seguiremos suponiendo que el sujeto de pago continúan siendo las empresas generadoras. Para asignar retiros a empresas generadoras es necesario conocer con quién está contratado cada cliente. La asignación de los retiros de cada barra a cada empresa generadora se hizo con información de contratos proporcionada por AES-Gener.

Ingresos tarifarios. En nuestros cálculos utilizamos los ingresos tarifarios estimados en CDEC-SIC (2003). Estos se calculan con los factores de penalización publicados por la CNE en el Informe de Precio de Nudo de octubre de cada año y se obtienen de la operación simulada del sistema. ${ }^{11}$

Los ingresos tarifarios calculados por el CDEC-SIC para 2002 (US $\$ 4,81$ millones) son distintos a los ingresos reales de energía y potencia por tramo: US $\$ 14,5$ millones. ${ }^{12}$ Estos son los ingresos que, en definitiva, determinan los peajes del sistema de transmisión efectivamente pagados. Los ingresos tarifarios y reales difieren, porque ingresos reales por tramo se obtienen de los costos marginales por barra calculados por el CDEC en la operación horaria real, mientras que los ingresos tarifarios calculados por el CDEC-SIC corresponden a la operación simulada y esperada.

En este trabajo utilizamos los ingresos tarifarios estimados por la Dirección de Peajes del CDEC-SIC, porque provienen de una fuente independiente y son ampliamente conocidos por quienes trabajan en el sector, además que reflejan condiciones esperadas.

\subsection{Modelación del Sistema de Transmisión}

El sistema de transmisión se agrega al modelo con factores de pérdidas óhmicas para la demanda total del sistema incluyendo el despacho obligado de la central Guacolda por mínimos técnicos.

\subsection{Cálculo de las Prorratas}

Como se dijo, para calcular la prorrata de cada línea excluimos a las centrales o cargas que participan con, según sea el caso, factores GGDF o GLDF negativos, pero fijamos el umbral de corte en $0 \%$, vale decir, toda central o carga con factor positivo participa en la prorrata. Además, es necesario especificar el criterio para seleccionar aquellas condiciones de operación que se usarán para calcular las

\footnotetext{
${ }^{11}$ Los ingresos tarifarios provienen de las diferencias de precios entre nudos que, a su vez, reflejan las pérdidas marginales de potencia. De acuerdo a la ley actual estos ingresos tarifarios pagan parte de la anualidad y los costos de mantención que le corresponde a un generador de acuerdo con su contrato; el resto lo paga con peajes básicos y adicionales.

12 Estos ingresos reales de energía y potencia son los atribuibles a las líneas del troncal. La fuente son los Balances de Energía y Potencia mensuales del CDEC-SIC. Transelec (2002) reconoce US\$26,2 millones de ingresos reales totales por todas sus instalaciones.
} 
prorratas. En este trabajo ocuparemos el criterio de los percentiles superiores y el criterio de los porcentajes del flujo máximo.

El Gráfico 2 muestra ambos criterios. Las 1.440 condiciones de operación dan origen a 1.440 flujos de potencia por cada línea. Para cada línea, el valor absoluto de los flujos se ordena de mayor a menor y las prorratas se calculan sólo con los flujos de las condiciones de operación seleccionadas. Con el criterio de los percentiles se seleccionan aquellas condiciones de operación con flujos en los percentiles superiores de la distribución. En el gráfico el umbral es el percentil 90; vale decir, se incluyen aquellas condiciones de operación tales que la magnitud de su flujo está en el 10\% superior de la distribución. En el trabajo simulamos los pagos para los percentiles $0,10,20$ y hasta 90 .

Con el criterio del porcentaje del flujo se seleccionan aquellas condiciones de operación con flujos iguales o mayores al $x \%$ del flujo máximo. En el gráfico $x=90$, lo que implica que para calcular las prorratas se incluyen aquellas condiciones de operación que causan flujos iguales o mayores que el $90 \%$ del flujo máximo. En el trabajo simulamos los pagos para $x=0,10,20, \ldots ., 90$.

Adicionalmente, estudiaremos el método propuesto por la CNE en enero de 2003 (al que llamamos CNE3). El prorrateo de este método es algo complicado, y por eso postergamos su discusión para la sección 5.2.

\section{GRAFICO 2}

CRITERIOS DE CORTE PARA EL CALCULO DE LAS PRORRATAS

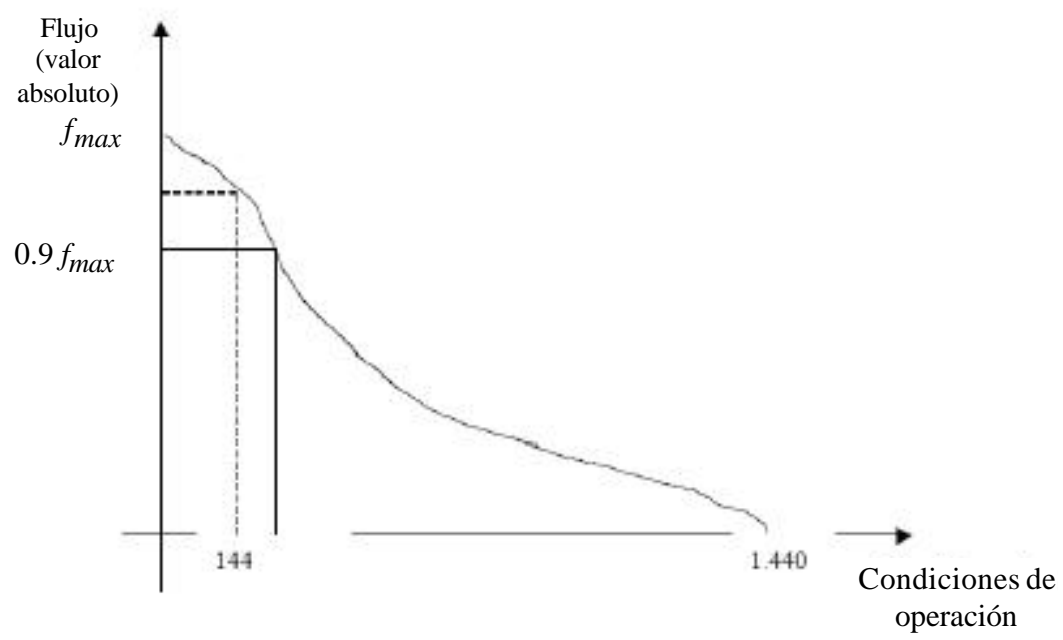




\section{Resultados}

\subsection{La Situación Inicial}

El Cuadro 2 muestra los ingresos de Transelec en 2001, descompuestos por empresa generadora y por categoría de ingreso (se incluyen todas las instalaciones, no sólo las del troncal). El 83,31\% de sus US\$137,39 millones de dólares de ingresos provino de las empresas del grupo Endesa (en adelante simplemente Endesa), el resto de Colbún, Gener y los restantes generadores (otros). ${ }^{13}$ Almismo tiempo, poco menos del $75 \%$ de los ingresos del transmisor provienen de peajes y poco más de un quinto de ingresos tarifarios.

El Cuadro 3 descompone los pagos de cada grupo de empresas en las distintas categorías. ${ }^{14}$ Gran parte de los peajes básicos corresponde, seguramente, a instalaciones que se incluyen en el troncal definido por la CNE. Los peajes adicionales, por otro lado, corresponden principalmente a líneas de inyección y de retiro y muy pocas forman parte del troncal.

Los ajustes necesarios para estimar los pagos de peaje por las instalaciones del troncal en 2001, según los contratos, se muestran en el Cuadro 4. La columna 1 reproduce los pagos de peaje básico estimados en el Cuadro 3 (columna 1). Para obtener los pagos por el troncal le sumamos a esta columna los pagos de peaje adicional que corresponden a instalaciones del troncal (columna 2), y le restamos los pagos de peaje básico por instalaciones que no están en el troncal (columna 3). El resultado de (1)+(2)-(3) es la columna 4, nuestra estimación del pago de peajes en 2001 por instalaciones del sistema troncal. De esta columna se deducen las prorratas de cada grupo generador (columna 5).

\section{CUADRO 2}

INGRESOS DE TRANSELEC, AÑO 2001

(en millones de dólares)

\begin{tabular}{lrr}
\hline Fuente de los ingresos & Ingresos & $\%$ \\
\hline Por empresa generadora & & \\
Colbún & 9,12 & 6,64 \\
Endesa & 114,46 & 83,31 \\
Gener & 7,50 & 5,46 \\
Otros & 6,31 & 4,59 \\
Total & 137,39 & 100 \\
& & \\
Por categoría de ingreso & & \\
Peaje básico & 73,27 & 53,3 \\
Peaje adicional & 28,30 & 20,6 \\
Ingresos tarifarios & 31,19 & 22,7 \\
Otros arriendos & 4,67 & 3,4 \\
Total & 137,39 & 100 \\
\hline
\end{tabular}

Fuente: Transelec (2001).

\footnotetext{
13 Véase el Cuadro A1 del Apéndice para un detalle de las empresas que componen cada grupo.

14 En el Apéndice explicamos cómo obtuvimos el Cuadro 3.
} 
CUADRO 3

ESTIMACION DE PAGOS POR GRUPO GENERADOR, AÑO 2001

(en millones de dólares)

\begin{tabular}{lrrrrr}
\hline & $\begin{array}{c}(1) \\
\text { Peaje } \\
\text { básico }\end{array}$ & $\begin{array}{c}\text { Peaje } \\
\text { adicional }\end{array}$ & $\begin{array}{c}(3) \\
\text { Ingresos } \\
\text { tarifarios }\end{array}$ & $\begin{array}{c}(4) \\
\text { Arriendos }\end{array}$ & $\begin{array}{c}\text { (5) } \\
\text { Total }\end{array}$ \\
\hline Colbún & 4,35 & 4,10 & 0,67 & - & 9,12 \\
Endesa & 63,98 & 22,42 & 28,06 & - & 114,46 \\
Gener & 4,70 & 1,78 & 1,03 & - & 7,50 \\
Otros & 0,20 & - & 1,43 & 4,67 & 6,31 \\
Total & 73,23 & 28,30 & 31,19 & 4,67 & 137,39 \\
\hline
\end{tabular}

Fuente: La fuente de los pagos de Colbún y Gener son las propisas compañías. El resto se calculó con datos de Transelec (2001) y el método y los supuestos explicados en el apéndice A.

Nota: Los ingresos tarifarios son aquellos que aparecen en la memoria de Transelec (2001) y no incluyen las reliquidaciones posteriores.

\section{CUADRO 4 \\ ESTIMACION DE PAGOS POR SISTEMA TRONCAL POR GRUPO GENERADOR, AÑO 2002}

(en millones de dólares)

\begin{tabular}{lcccrrr}
\hline & $\begin{array}{c}(1) \\
\text { Peaje } \\
\text { básico } \\
\text { total }\end{array}$ & $\begin{array}{c}(2) \\
\text { Peaje } \\
\text { adicional } \\
\text { troncal }\end{array}$ & $\begin{array}{c}\text { Peajes } \\
\text { básicos no } \\
\text { troncal }\end{array}$ & $\begin{array}{c}\text { Pagos por } \\
\text { troncal } \\
(2001)\end{array}$ & $\begin{array}{c}(5) \\
\text { Prorrata }\end{array}$ & $\begin{array}{c}(6) \\
\text { Pagos por } \\
\text { troncal } \\
(2002)\end{array}$ \\
\hline Colbún & 4,35 & 1,35 & - & 5,70 & $11,34 \%$ & 8,95 \\
Endesa & 63,98 & - & 24,59 & 39,39 & $78,35 \%$ & 61,86 \\
Gener & 4,70 & 0,47 & 0,19 & 4,98 & $9,91 \%$ & 7,82 \\
Otros & 0,20 & - & - & 0,20 & $0,4 \%$ & 0,32 \\
Total & 73,23 & 1,82 & 24,59 & 50,27 & $100,0 \%$ & 78,95 \\
\hline
\end{tabular}

Fuente: La columna 1 corresponde a la columna del Cuadro 3.

La columna 2 son los peajes adicionales atribuibles a líneas que forman parte del troncal. Sólo teníamos información para Colbún y Gener y por eso suponemos que Endesa no pagó peajes adicionales por líneas del sistema troncal en 2001. Nótese que este supuesto tiende a disminuir la prorrata de Endesa.

La columna 3 incluye los peajes pagados por líneas que no forman parte del troncal. En su gran mayoría se trata de líneas de inyección declaradas por Endesa. Para calcular la prorrata de Endesa en el sistema troncal en 2001 hemos supuesto que ninguna línea de inyección de Endesa forma parte del sistema troncal. Este supuesto también tiende a disminuir la prorrata de Endesa.

La columna 4 estima los pagos por líneas del troncal en 2001 según los contratos vigentes entre generadores y Transelec en 2001. Es igual a (1) + (2) - (3).

La columna 5 es la prorrata que le correspondió a cada grupo de generadores en 2001, según los peajes de la columna 4.

Finalmente, la columna 6 reparte los peajes por el sistema troncal estimados para 2002 (78,95 millones de dólares) de acuerdo con las prorratas de la columna 5. La columna 6 se traspasa a la columna 1 del Cuadro 5.

Es importante notar que hemos supuesto que Endesa no paga peajes adicionales por instalaciones del troncal (columna 2). Además, hemos supuesto que 
ninguna línea de inyección de Endesa forma parte del sistema troncal: Endesa declaró pagar US\$24,59 millones por estas líneas, los que aparecen en la columna 3. Ambos supuestos tienden a subestimar la prorrata de Endesa. Elegimos sesgar nuestros errores en esa dirección, porque, como anticipamos en la introducción, nuestro principal resultado es que los métodos propuestos tienden a disminuir el pago de peajes de Endesa. Esta disminución es menor mientras menores sean los peajes actuales y, por lo tanto, nuestro resultados entregan una cota inferior de esta disminución.

Por último, del informe de peajes (CDEC-SIC [2003]) obtenemos el (AVNR+COyM) del sistema troncal (véase el Cuadro A2 en el Apéndice para el desglose línea por línea) y los ingresos tarifarios (IT), que ascienden a US $\$ 4,81$ millones de dólares. La diferencia (AVNR+COyM-IT) determina los peajes estimados para el troncal en 2002, US\$78,95 millones. Estos se prorratean de acuerdo a la columna 5 del Cuadro 4 y el resultado es la columna 6, nuestra estimación del pago de peajes por el sistema troncal en 2002. Esta columna se traspasa al Cuadro $3 .{ }^{15}$

Nuestra estimación es que en 2002 Colbún pagó poco más del 11\% de los US $\$ 78,95$ millones de peajes atribuibles al troncal; Endesa $79 \%$ y Gener el $10 \%$ restante. Gran parte del sistema está siendo pagada por Endesa, lo que se debe a que sus centrales, en su mayoría hidráulicas, tienden a ubicarse alejadas de Santiago, el principal centro de consumo. Por ello, sus áreas de influencia tienden a ser extensas.

\subsection{Peajes con Distintas Reglas de Asignación de Uso}

El punto de referencia de nuestros ejercicios. El Cuadro 5 muestra los pagos que hubieran hecho en 2002 las principales empresas generadoras bajo distintas reglas de asignación. El punto de referencia de nuestros ejercicios es la columna 1 ("Pagos según contratos"), nuestra estimación del pago de peajes según contratos.

Inyecciones y retiros: peajes calculados con GGDF y GLDF. Las columnas 2 y 3 prorratean los peajes usando los factores GGDF y GLDF respectivamente. Cabe notar que en este cuadro reportamos las simulaciones usando el criterio de los percentiles y suponiendo que para calcular las prorratas se consideran las 1.440 condiciones de operación. Como ya se discutió latamente, los factores GGDF atribuyen los flujos de una línea a las inyecciones que hacen las centrales, en tanto que los factores GLDF los atribuyen a los retiros. Por lo tanto, la columna 2 es la regla $100-0$, en el sentido que le asigna el $100 \%$ del uso de una línea a las inyecciones. Por el contrario, la columna 3 es la regla $0-100$, porque le asigna el $100 \%$ del uso de una línea a quienes retiran. Por supuesto, la asignación de peajes que aparece en la columna 3 supone que el sujeto de pago son las empresas generadoras, quienes pagarían por cuenta del uso de líneas que hagan sus clientes.

15 Como ya se dijo, en marzo de 2002 vencieron varios acuerdos de peaje, y algunos todavía no se han renovado. Por eso es más representativo el pago efectivo de peajes de 2001 . 


\section{CUADRO 5 \\ PAGOS DE PEAJE, AÑO 2002 \\ SUJETO DE PAGO: GRUPOS GENERADORES \\ (en millones de dólares)}

\begin{tabular}{|c|c|c|c|c|c|c|c|}
\hline & $\begin{array}{c}(1) \\
\text { Pagos } \\
\text { según } \\
\text { contratos }\end{array}$ & $\begin{array}{c}(2) \\
\text { GGDF } \\
100-0\end{array}$ & $\begin{array}{c}(3) \\
\text { GLDF } \\
0-100\end{array}$ & $\begin{array}{c}(4) \\
\text { CNE } 1 \\
50-50 \\
(05 / 2002)\end{array}$ & $\begin{array}{c}(5) \\
\text { CNE } 2 \\
50-50 \\
(12 / 2002\end{array}$ & $\begin{array}{c}(6) \\
\text { CNE } 3 \\
(01 / 2003)\end{array}$ & $\begin{array}{c}(7) \\
\text { Método } \\
\text { CDEC }\end{array}$ \\
\hline Colbún & 8,95 & 9,94 & 7,89 & 4,97 & 8,91 & 9,70 & 4,54 \\
\hline Endesa & 61,86 & 53,31 & 35,53 & 26,65 & 44,42 & 42,25 & 53,19 \\
\hline Gener & 7,82 & 7,72 & 28,67 & 3,86 & 18,20 & 18,05 & 12,49 \\
\hline Otros & 0,32 & 7,99 & 6,87 & 3,99 & 7,43 & 8,96 & 8,73 \\
\hline Total peajes & 78,95 & 78,95 & 78,95 & 39,47 & 78,95 & 78,95 & 78,95 \\
\hline IT & 4,81 & 4,81 & 4,81 & 4,81 & 4,81 & 4,81 & 4,81 \\
\hline $\mathrm{AVNR}+\mathrm{COyM}$ & 83,76 & 83,76 & 83,76 & 83,76 & 83,76 & 83,76 & 83,76 \\
\hline
\end{tabular}

La columna 2 indica que, comparado con los contratos actuales, esta regla reduce el pago de Endesa en US\$8,55 millones, que se redistribuyen casi totalmente al grupo de "otros" generadores, quienes aumentarían su pago de peajes desde apenas US\$320.000 a casi US\$8 millones.

La distribución de pagos hubiese sido muy distinta si el uso del sistema se hubiera determinado por los retiros. En ese caso la cuenta de Endesa hubiera caído casi a la mitad (desde US\$61,86 millones a US\$35,53). Por contraste, el grupo Gener hubiera pagado US\$28,67 millones, cuatro veces más que los US\$7,82 millones bajo los contratos actuales. Colbún y el resto de los generadores hubieran pagado montos similares a los determinados según GGDF.

Nótese que los peajes presentados en la columna 3 suponen que los sujetos de pago por retiros son las empresas generadoras, quienes, a su vez, tienen contratos con los clientes que retiran. Como ya se dijo, en un mercado competitivo los precios son libres y los nuevos contratos reflejarían el peaje de cada cliente que contrata con una empresa generadora. Siendo este peaje el mismo, independientemente de la empresa que lo surta, el efecto no debiera afectar el balance de cada empresa generadora. El cálculo reportado en la columna 3, por tanto, es una indicación del pago que hubiera hecho cada generador por cuenta de los clientes que tenía contratados en 2002 en un equilibrio de largo plazo. Sin embargo, como se dijo, en el largo plazo la incidencia de tal cargo debiera ser, tal que todos los peajes serían pagados por los consumidores.

Los dos 50-50 de la CNE (mayo y diciembre de 2002). Las columnas 2 y 3 son útiles, porque muchas reglas de asignación son promedios ponderados de las columnas 2 y 3 . Así, la columna 4 resume lo que hubiera debido pagar cada empresa con la propuesta original de la ley corta (a la que, por brevedad, denotamos por CNE1). En el proyecto enviado al Congreso en mayo de 2002 el $50 \%$ de los peajes se hubiera asignado por inyecciones y el $50 \%$ por retiros. Sin embargo, el sujeto de pago de los retiros hubiese sido cada cliente, no la empresa generadora con quien tiene contrato (vale decir, en términos del Gráfico 1 las celdas relevantes 
hubieran sido la $(1,1)$ y la $(2,2))$. El transmisor le hubiera cobrado directamente a los clientes. Se aprecia en la columna 4 que, en ese caso, el pago directo de Colbún, Gener y Endesa hubiera caído a más o menos la mitad. La caída de los peajes de Endesa es, obviamente, la mayor: US\$ 35,21 millones (desde US\$61,86 millones a US $\$ 26,65$ millones).

La columna 5 indica los pagos que le hubieran correspondido a cada generador en 2002 con la propuesta de la CNE materializada en una indicación al proyecto de ley introducida en diciembre de 2002 (a la que, por brevedad, denotamos CNE2). Tal como ocurre con CNE1, esta indicación le asignaba el 50\% de los peajes a las inyecciones y el $50 \%$ a los retiros. Sin embargo, en este caso el sujeto de pago eran los generadores - de ahí que a veces se dijera que esta regla de asignación era un $100-0,100 \%$ a los generadores $0 \%$ a los clientes-. Para obtener los pagos, entonces, simplemente promediamos las columnas 2 y 3 .

Conociendo las columnas 2 y 3 , ya no sorprende que el pago de Endesa disminuya en poco más de US\$17 millones (desde US\$61,86 millones a US $\$ 44,42$ millones), y que Gener los aumente en poco más de US $\$ 10$ millones (desde US $\$ 7,82$ millones a US $\$ 18,20$ millones). Pero es interesante comparar las columnas 4 y 5 , que difieren fuertemente. En esencia, con la regla CNE1 se redistribuyen peajes desde los tres principales grupos generadores hacia los consumidores. Con la regla CNE2 la redistribución es desde Endesa hacia Gener y los otros generadores, mientras que el pago de Colbún es muy parecido.

Por supuesto, como ya se dijo, la incidencia de ambas reglas sería la misma si los peajes se traspasaran competitivamente a los consumos. Sin embargo, en la práctica existen contratos preexistentes. Si esto es así, el peaje de un generador dado sólo puede bajar si el resto de los generadores soporta la pérdida de capital correspondiente.

La propuesta de enero de 2003. En enero de 2003 la CNE propuso determinar los peajes con un nuevo método. Aunque esta propuesta ya se descartó, es interesante analizarla y cuantificarla, porque, como se verá, sugiere que métodos en apariencia muy distintos pueden llevar a pagos cuantitativamente muy similares.

El método combinaba cobros por inyecciones y retiros de la siguiente forma.

- $\quad$ Para cada carga $c$ se calcula su prorrata en cada uno de los tramos del sistema troncal promediándose las distintas condiciones de operación. Estas prorratas se ordenan de mayor a menor, determinándose la máxima prorrata de la carga $c$, llamémosla (prorrata máxima ${ }_{c}$ ).

- $\quad$ En todo tramo tal que la prorrata de la carga $c$ es igual o mayor que $0,7 \times\left(\right.$ prorrata máxima $\left._{c}\right)$, a la empresa generadora que tenga contrato con la carga $c$ le corresponde pagar un porcentaje igual a la prorrata de la carga. $\mathrm{Si}$ es menor que $0,7 \times\left(\right.$ prorrata máxima $\left.{ }_{c}\right)$, entonces la empresa generadora no paga nada por cuenta de la carga $c$ en ese tramo. 
Por ejemplo, si (prorrata máxima $\left.{ }_{c}\right)=0,45$, entonces la empresa generadora que tenga contrato con $c$ tendrá que pagar por cuenta de $c$ en cada tramo donde la prorrata de $c$ sea igual o mayor que $0,7 \times 0,45=0,315$. Si la prorrata de $c$ en el tramo $l \rightarrow k$ es, por ejemplo, 0,1 , entonces la empresa generadora no paga peaje por cuenta de $c$ en ese tramo. Por otro lado, si la prorrata de $c$ fuera 0,38 , la empresa generadora debe pagar el $38 \%$ del peaje que le corresponde a ese tramo.

Para cada tramo el ejercicio determinará un conjunto de cargas que pagarán y la suma de pagos recibidos por el transmisor, llamémosla (pagos por retiros p $_{l \rightarrow k}$ ). Esta cantidad será menor que el peaje de $l \rightarrow k, \pi_{l \rightarrow k}$, porque algunas cargas con factores GLDF positivos quedarán exentas de pago. La diferencia

$$
\pi_{l \rightarrow k}-\left(\text { pagos por retiros }_{l \rightarrow k}\right)
$$

se le cargará directamente a los generadores, según las inyecciones de las centrales de cada uno, de la siguiente forma:

- $\quad$ En analogía con las cargas, para cada central generadora $g$ se calcula su prorrata en cada uno de los tramos del sistema troncal. Estas prorratas se ordenan de mayor a menor determinándose (prorrata máxima ${ }_{g}$ ).

- $\quad$ En todo tramo tal que la prorrata de central generadora $g$ es igual o mayor que $0,1 \times\left(\right.$ prorrata máxima $\left._{g}\right)$, a la empresa generadora le corresponde pagar un porcentaje igual a la prorrata de la central. Si es menor que $0,1 \times($ prorrata máxima $_{c}$ ), entonces la empresa generadora no paga nada por cuenta de la central $g$ en ese tramo. En otras palabras, $10 \%$ de la prorrata máxima es el umbral de corte de la central $g$ en cada tramo.

- $\quad$ Para todo tramo, y una vez excluidas aquellas centrales con prorratas menores que su umbral de corte, el resto de las prorratas se escala para que sumen uno.

- $\quad$ El pago por peaje del tramo $l \rightarrow k$ atribuido a la central $g$ corresponde, entonces, a su prorrata corregida multiplicada por la diferencia $\pi_{l \rightarrow k}-($ pagos por retiros r $\rightarrow k$ ).

El Cuadro 6 muestra el resultado producido por este método. Como se aprecia en el Cuadro 6 el 58,86\% de los pagos se atribuye a inyecciones y el 41,14\% a retiros. Sin embargo, cuando ambos se suman, se comprueba que los pagos por empresa son notablemente parecidos al promedio simple de los GGDF y GLDF (ver las columnas 4 y 5 del Cuadro 3).

¿Por qué ocurre que con un método en apariencia tan distinto al promedio simple de los GGDF y GLDF se llega resultados tan similares? El punto de fondo es que los grados de libertad son muchos, porque el pago final de cada agente es el resultado de varios parámetros y reglas: el umbral de corte, el percentil de las condiciones de operación a partir del cual se calculan las prorratas, la ponderación de inyecciones y de retiros, etc. La lección más general es que, independientemen- 
te del método que se seleccione para prorratear los peajes, el valor de los parámetros es parte central y no accesoria de la regulación de transmisión. Si estos parámetros no se fijan transparentemente, la autoridad que reciba el poder de fijarlos podrá, en principio, distribuir los peajes entre distintos agentes casi de cualquier manera.

\begin{tabular}{cccc} 
CUADRO 6 \\
DISTRIBUCION DE PEAJES AÑO 2002 CON EL MET \\
CNE DE ENERO DE 2003 (CNE3) \\
(en millones de dólares) \\
\hline \multicolumn{4}{c}{$(1)$} \\
Inyecciones & Retiros & Total \\
\hline Colbún & 6,25 & 3,45 & 9,70 \\
Endesa & 29,97 & 12,28 & 42,25 \\
Gener & 5,15 & 12,90 & 18,05 \\
Otros & 5,10 & 3,85 & 8,96 \\
Total & 46,47 & 32,48 & 78,95 \\
& $58,86 \%$ & $41,14 \%$ \\
\hline
\end{tabular}

Nota: Este cuadro desglosa la columna 6 del Cuadro 3.

\subsection{Robustez y Sensibilidad a los Supuestos}

La principal conclusión del análisis precedente es que la magnitud de la redistribución de peajes depende fuertemente de la ponderación que reciban los retiros. Sin embargo, los resultados presentados en el Cuadro 5 se obtuvieron calculando las prorratas con las 1.440 condiciones de operación ¿Qué tan sensibles son los resultados si las prorratas se calculan con sólo una fracción de las condiciones de operación?

\section{CUADRO 7}

DISTRIBUCION DE PEAJES DE ACUERDO AL INFORME DE PEAJES DEL CDEC-SIC, 2002

(en millones de dólares)

\begin{tabular}{lccr}
\hline & $\begin{array}{c}(1) \\
\text { Peaje básico }\end{array}$ & $\begin{array}{c}(2) \\
\text { Peaje adicional }\end{array}$ & $\begin{array}{c}(3) \\
\text { Total }\end{array}$ \\
\hline Colbún & 3,54 & 1,00 & 4,54 \\
Endesa & 52,92 & 0,27 & 53,19 \\
Gener & 8,92 & 3,57 & 12,49 \\
Otros & 6,47 & 2,26 & 8,73 \\
Total & 71,85 & 7,10 & 78,95 \\
& $91,01 \%$ & $8,99 \%$ & $100 \%$ \\
\hline
\end{tabular}

Nota: Este cuadro desglosa la columna 7 del Cuadro 3. Los peajes se calcularon aplicando la Resolución Ministerial No 52 de 2002 del Ministerio de Economía. Es importante notar que esta resolución fue impugnada por Endesa y Guacolda. 
Los Cuadros 8, 9 y 10 muestran que los pagos por grupos de empresas son muy estables. El Cuadro 8 muestra los pagos de cada empresa generadora si las prorratas de cada línea se calculan usando sólo las 144 condiciones de operación con mayor flujo (el decil 1), las 288 condiciones de operación con mayor flujo (el decil 2) y así sucesivamente hasta la columna 10, que es equivalente a la columna 3 del Cuadro 3. Como se aprecia, la variación de los pagos es muy pequeña. El Cuadro 9 indica que los pagos también serían muy similares si se utilizara el criterio del flujo máximo, independientemente del porcentaje del flujo máximo que se elija.

\section{CUADRO 8 \\ PAGOS POR GRUPO EMPRESARIAL METODO GGDF, CRITERIO DE LOS PERCENTILES} (en millones de dólares)

\begin{tabular}{|c|c|c|c|c|c|c|c|c|c|c|}
\hline & 1 & 2 & 3 & 4 & 5 & 6 & 7 & 8 & 9 & 10 \\
\hline Colbún & 11,24 & 10,97 & 10,81 & 10,50 & 10,44 & 10,28 & 10,16 & 10,08 & 9,95 & 9,94 \\
\hline Endesa & 54,76 & 54,76 & 54,65 & 54,51 & 54,46 & 54,37 & 54,12 & 54,00 & 53,83 & 53,31 \\
\hline Gener & 6,10 & 6,18 & 6,31 & 6,63 & 6,62 & 6,81 & 7,12 & 7,18 & 7,30 & 7,72 \\
\hline Otros & 6,85 & 7,04 & 7,18 & 7,30 & 7,44 & 7,50 & 7,55 & 7,69 & 7,87 & 7,99 \\
\hline Total & 78,95 & 78,95 & 78,95 & 78,95 & 78,95 & 78,95 & 78,95 & 78,95 & 78,95 & 78,95 \\
\hline
\end{tabular}

Nota: 1 indica que para calcular las prorratas se consideró sólo el 10\% de los flujos mayores (144 condiciones de operación).

\section{CUADRO 9 \\ PAGOS POR GRUPO EMPRESARIAL CRITERIO DE LOS PORCENTAJES DEL FLUJO MAXIMO}

(en millones de dólares)

\begin{tabular}{lrrrrrrrrrr}
\hline & $90 \%$ & $80 \%$ & $70 \%$ & $60 \%$ & $50 \%$ & $40 \%$ & $30 \%$ & $20 \%$ & $10 \%$ & $0 \%$ \\
\hline Colbún & 10,90 & 11,19 & 10,84 & 10,90 & 10,66 & 10,47 & 10,27 & 10,18 & 10,08 & 9,94 \\
Endesa & 54,79 & 55,13 & 55,30 & 54,79 & 54,59 & 54,45 & 54,34 & 53,81 & 53,65 & 53,31 \\
Gener & 6,23 & 6,22 & 6,08 & 6,23 & 6,55 & 6,60 & 6,81 & 7,16 & 7,25 & 7,72 \\
Otros & 7,03 & 6,41 & 6,73 & 7,03 & 7,15 & 7,43 & 7,54 & 7,80 & 7,96 & 7,99 \\
Total & 78,95 & 78,95 & 78,95 & 78,95 & 78,95 & 78,95 & 78,95 & 78,95 & 78,95 & 78,95 \\
\hline
\end{tabular}

Nota: $90 \%$ indica que para calcuar las prorratas se consideraron sólo aquellos flujos mayores o iguales que el $90 \%$ del flujo máximo de la línea. $0 \%$ incluye las 1.440 condiciones de operación. 


\section{CUADRO 10 \\ PAGOS POR RETIROS}

METODO DE LOS GLDF, CITERIO DE LOS PERCENTILES

(en millones de dólares)

\begin{tabular}{lrrrrrrrrrr}
\hline & \multicolumn{1}{c}{2} & \multicolumn{1}{c}{3} & \multicolumn{1}{c}{4} & \multicolumn{1}{c}{5} & \multicolumn{1}{c}{6} & \multicolumn{1}{c}{7} & \multicolumn{1}{c}{8} & 9 & 10 \\
\hline Colbún & 8,14 & 8,10 & 7,94 & 7,75 & 7,55 & 7,48 & 7,46 & 7,55 & 7,75 & 7,89 \\
Endesa & 34,15 & 34,03 & 34,21 & 34,54 & 34,84 & 35,00 & 35,21 & 35,23 & 35,25 & 35,53 \\
Gener & 28,17 & 28,49 & 28,74 & 29,00 & 29,12 & 29,25 & 29,24 & 29,19 & 29,07 & 28,67 \\
Otros & 8,48 & 8,32 & 8,06 & 7,65 & 7,44 & 7,22 & 7,05 & 6,98 & 6,88 & 6,87 \\
Total & 78,95 & 78,95 & 78,95 & 78,95 & 78,95 & 78,95 & 78,95 & 78,95 & 78,95 & 78,95 \\
\hline
\end{tabular}

Sin embargo, la aparente estabilidad esconde que el pago de peajes de algunas centrales varía fuertemente, dependiendo de qué percentil se elija. El Cuadro 11 muestra los pagos de seis centrales. Se puede apreciar que los peajes de El Toro, Nehuenco o Nueva Renca varían muy poco, pero no así los de Canutillar, Colbún o Guacolda. Considérese el caso de Canutillar. Si, como se hizo para computar la columna 3 del Cuadro 5, se usan las 1.440 hidrologías para computar su prorrata, le hubiera correspondido pagar un peaje de US\$7,54 millones. Por contraste, si para calcular las prorratas se ocupan únicamente las 144 condiciones de operación con mayores flujos, el peaje de Canutillar caería casi $30 \%$ a US $\$ 5,45$ millones. Por contraste, la central Colbún hubiera pagado más si se hubieran considerado sólo las 144 condiciones de operación con mayores flujos: US\$4,48 millones contra US $\$ 3,08$ millones. Por último, la variación del peaje de la central Guacolda también es importante: US\$1 millón contra US\$3,15 millones. Nótese que variaciones de US\$1 o US\$2 millones son considerables como porcentaje de la inversión en centrales, toda vez que se trata de pagos anuales.

\section{CUADRO 11 \\ PAGOS DE CENTRALES SELECCIONADAS METODO GGDF, CRITERIO DE LOS PERCENTILES}

(en millones de dólares)

\begin{tabular}{|c|c|c|c|c|c|c|c|c|c|c|}
\hline & 1 & 2 & 3 & 4 & 5 & 6 & 7 & 8 & 9 & 10 \\
\hline Canutillar & 5,45 & 5,56 & 5,96 & 6,48 & 6,89 & 7,21 & 7,48 & 7,57 & 7,53 & 7,54 \\
\hline Colbún & 4,48 & 4,25 & 4,10 & 3,88 & 3,76 & 3,57 & 3,41 & 3,27 & 3,13 & 3,08 \\
\hline El Toro & 10,72 & 10,60 & 10,42 & 10,39 & 10,55 & 10,83 & 11,18 & 11,50 & 11,72 & 11,65 \\
\hline Guacolda & 1,00 & 1,12 & 1,38 & 1,84 & 1,84 & 2,12 & 2,52 & 2,63 & 2,84 & 3,15 \\
\hline Nehuenco & 2,53 & 2,60 & 2,64 & 2,60 & 2,66 & 2,69 & 2,70 & 2,73 & 2,74 & 2,86 \\
\hline Nueva Renca & 2,64 & 2,63 & 2,59 & 2,51 & 2,50 & 2,47 & 2,43 & 2,39 & 2,35 & 2,42 \\
\hline Resto & 52,14 & 52,18 & 51,87 & 51,24 & 50,75 & 50,06 & 49,23 & 48,85 & 48,65 & 48,25 \\
\hline Total & 78,95 & 78,95 & 78,95 & 78,95 & 78,95 & 78,95 & 78,95 & 78,95 & 78,95 & 78,95 \\
\hline
\end{tabular}


Si bien gran parte de estas variaciones se cancela a nivel de grupos, las decisiones de localización se toman a nivel de cada central. En vista de que el criterio de corte generalmente se determina por reglamento, esto sugiere que las señales de localización del método GGDF se determinarán discrecionalmente sin que sean eficientes. En segundo lugar, nótese que la variabilidad de los peajes de cada central implica que asume más riesgo una empresa pequeña y, por lo tanto, favorece la concentración del sector de generación.

\section{Conclusiones}

Dijimos en la introducción que el propósito de este trabajo era estudiar qué tan sensible es el pago de peajes a las reglas de asignación y los parámetros necesarios para implementarla. La conclusión es que la distribución de peajes determinada por cada regla de asignación depende, por lo menos, de tres parámetros: (i) el porcentaje de las condiciones de operación que se ocupa para calcular las prorratas; (ii) el umbral de corte; (iii) la ponderación que se le asigne a los retiros y las inyecciones. Como lo sugiere el tercer método propuesto por la CNE (CNE3), quien esté encargado de fijar el valor de los parámetros necesarios para materializar el método tendría amplios grados de libertad. Por eso, el valor de los parámetros es parte central y no accesoria de la regulación de transmisión. Si estos parámetros no se fijan transparentemente, la autoridad que reciba el poder de fijarlos podría, en principio, distribuir los peajes entre distintos agentes casi de cualquier manera. Nuestros ejercicios también sugieren que el peaje de algunas centrales puede variar considerablemente dependiendo del nivel en que se fijen los tres parámetros. Esto implica que el costo de desarrollar proyectos intensivos en transmisión no será determinado por el método, sino por el nivel de los parámetros. Así, un mismo método puede dar señales de localización muy distintas.

Esta indeterminación permite poner en perspectiva el rol que pueden determinar los factores GGDF y GLDF en la regulación de peajes. La indeterminación indica que, seguramente, estos métodos permiten seleccionar parámetros que inducen pagos de peajes económicamente sensatos y dan señales económicas apropiadas. Pero, es importante notarlo, el marco conceptual que determina lo que es "económicamente apropiado" es anterior a los métodos GGDF o GLDF y debe definirse antes de fijar los parámetros específicos. En otras palabras, estos métodos pueden ser apropiados para prorratear los peajes de una línea determinada, pero sólo si antes se ha decidido quién debiera pagarlos de acuerdo con la lógica económica del problema. Por el contrario, son inadecuados para seleccionar, entre todos los agentes que participan del sistema, quién debe pagar una línea determinada.

El proyecto de ley corta que actualmente se discute en el Congreso (noviembre de 2003) se hace cargo en buena medida de estos problemas, y restringe la posterior discreción del regulador fijando reglas de asignación explícitas que indican cómo se debe determinar la participación de inyecciones y retiros en cada 
tramo del sistema de transmisión. ${ }^{16}$ Sin embargo, para evitar la discreción, es importante que las reglas específicas que deban precisarse en reglamentos y resoluciones sean discutidas y fundamentadas transparentemente.

\section{REFERENCIAS}

CDEC-SIC (2003), Informe de peajes, enero. Santiago: CDEC-SIC.

Comisión Nacional de Energía, CNE (2001), "Informe Técnico Definitivo: Fijación de Precios de Nudo, Sistema Interconectado Central (SIC) ", Santiago, mimeo.

Comisión Nacional de Energía (2002), "Propuesta del Ejecutivo, Sistema Transmisión Troncal SIC", mimeo, Santiago.

Comisión Nacional de Energía (2003), "Propuesta de Asignación de Pagos de Peajes en Sistema Troncal", mimeo, Santiago.

Díaz, C., A. Galetovic y R. Soto (2000), "La Crisis Eléctrica de 1998-1999: Causas, Consecuencias y Lecciones”, Estudios Públicos 80: 149-152.

Galetovic, A. y C. Muñoz (2004), “Transmisión Eléctrica y la 'Ley Corta': Una Estimación del Cargo Directo que Pagarán los Consumidores", Puntos de Referencia 273, Santiago: Centro de Estudios Públicos.

Galetovic, A., J. C. Olmedo y H. Soto (2002). “¿Qué tan Probable es una Crisis Eléctrica?”, Estudios Públicos 87: 175-212.

Galetovic, A. y R. Palma (2003), “Transmisión Eléctrica y la 'Ley Corta': Una Estimación de la Redistribución de Peajes”, Documento de Trabajo N 160, Centro de Economía Aplicada, Universidad de Chile.

Ng, W. (1981), "Generalized Generation Distribution Factors for Power System Security Evaluations", IEEE Transactions on Power Apparatus and System, Vol. PAS-100, 3: 1001-1005.

Olmedo, J. C. (2001), "El modelo GOL”, mimeo personal.

Rudnick, H., R. Palma y J. Fernández (1995), "Marginal Pricing and Suplement Cost Allocation in Transmission Open Access", IEEE Transactions on Power Systems 10: 1125-1142.

Transelec (2001), "Memoria Anual". Transelec, Santiago.

Transelec (2002), "Memoria Anual". Transelec, Santiago.

${ }^{16}$ Un análisis de este proyecto se encuentra en Galetovic y Muñoz (2004). 


\section{APENDICE}

\section{Pago de peajes en 2001}

El Cuadro 1 muestra los ingresos de Transelec en 2001. La memoria de Transelec detalla los montos que recibió de cada empresa generadora (parte superior del cuadro) y también los montos que recibió por cada categoría de ingresos -peajes básicos, adicionales, ingresos tarifarios y otros arriendos- (parte inferior del cuadro). Sin embargo, no descompone los pagos de cada grupo de generadores en estas cuatro categorías de ingreso, dato que necesitamos para estimar cuánto paga cada empresa actualmente por usar el sistema troncal.

Colbún y Gener nos entregaron el monto que pagaron en cada categoría durante 2001, y que aparece en el Cuadro 2. Para descomponer el pago de Endesa y de otros generadores, resolvimos el sistema de ecuaciones compuesto por: (i) cuatro ecuaciones que descomponen los pagos totales de, respectivamente, Endesa (E), Gener (G), Colbún (C) y otros generadores $(\mathrm{O})$ :

$$
P B_{i}+P A_{i}+I T_{i}+A_{i}=P T_{i}
$$

donde PB denota "peaje básico", PA "peaje adicional”, IT "ingreso tarifario", A "arriendo", PT "pago total"; (ii) y cuatro ecuaciones que descomponen los ingresos (I) que recibe Transelec () en cada categoría: peajes básicos, adicionales, ingresos tarifarios y otros arriendos.

$$
\begin{aligned}
& P B_{E}+P B_{G}+P B_{C}+P B_{O}=I P B_{T}, \\
& P A_{E}+P A_{G}+P A_{C}+P A_{O}=I P A_{T}, \\
& I T_{E}+I T_{G}+I T_{C}+I T_{O}=I I T_{T}, \\
& A_{E}+A_{G}+A_{C}+A_{O}=I A_{T},
\end{aligned}
$$

Sin embargo, la información con que contamos nos deja con más incógnitas que ecuaciones. Para completar el sistema de ecuaciones suponemos lo siguiente:

- Endesa no pagó “otros arriendos". Luego, por diferencia, los “otros arriendos" fueron pagados por los otros generadores.

- $\quad$ Se supone que los otros generadores pagan el 4,59\% de los ingresos tarifarios totales (US\$1.431 millones), la misma participación que tienen en los ingresos totales de Transelec.

El resultado de los cálculos es el Cuadro 2a. 
CUADRO A1

EMPRESAS Y GRUPOS

\begin{tabular}{ll}
\hline Empresa & Grupo \\
\hline Colbún & Colbún \\
Endesa & Endesa \\
Pangue & Endesa \\
Pehuenche & Endesa \\
San Isidro & Endesa \\
& \\
Energía Verde & Gener \\
ESSA & Gener \\
Gener & Gener \\
Guacolda 1 & Gener \\
& \\
Aconcagua & Otros \\
Arauco & Otros \\
Capullo & Otros \\
Fallas & Otros \\
HGV & Otros \\
Ibener & Otros \\
Morros & Otros \\
Petropower & Otros \\
Pullinque & Otros \\
Puntilla & Otros \\
Puyehue & Otros \\
S. Andes & Otros \\
Canalistas del Maipo & Otros \\
Petropower & Otros \\
\hline
\end{tabular}


CUADRO A2

EL SISTEMA TRONCAL

(Según la definición de la CNE en enero de 2003)

\begin{tabular}{|c|c|c|c|c|c|}
\hline Barra inicial & Barra final & Tensión & $\begin{array}{l}\text { AVNR + } \\
\text { CoyM }\end{array}$ & $\begin{array}{l}\text { Ingreso } \\
\text { tarifario }\end{array}$ & Peaje \\
\hline Jahuel & Ancona & 500 & $8.491,0$ & $1.146,0$ & $7.345,0$ \\
\hline Jahuel & Ancona & 500 & $9.713,0$ & $1.255,0$ & $8.458,0$ \\
\hline Maitencillo & Pan de Azúcar & 220 & $2.496,0$ & $-500 ., 0$ & $2.996,0$ \\
\hline Maitencillo & Pan de Azúcar & 220 & $2.496,0$ & $-500,0$ & $2.996,0$ \\
\hline B. Blancos & Puerto Montt & 220 & $1.467,0$ & $1.263,0$ & 204,0 \\
\hline Cardones & Maitencillo & 220 & $2.245,0$ & $-1.401,0$ & $3.646,0$ \\
\hline Cardones & Maitencillo & 220 & $2.434,0$ & $-1.401,0$ & $3.835,0$ \\
\hline Ancoa & Charrúa & 220 & $5.170,5$ & 769,5 & $4.401,0$ \\
\hline Ancoa & Charrúa & 220 & $5.170,5$ & 769,5 & $4.401,0$ \\
\hline Charrúa & Esperanza & 220 & $1.610,0$ & $1.859,0$ & $-249,0$ \\
\hline Chena & Jahuel & 220 & 462,0 & 776,0 & $-314,0$ \\
\hline Chena & Jahuel & 220 & 462,0 & 773,0 & $-311,0$ \\
\hline D. de Almagro & Carrera Pinto & 220 & $1.514,0$ & $2.203,0$ & $-689,0$ \\
\hline Esperanza & Victoria & 220 & 909,5 & $-861,0$ & $1.770,5$ \\
\hline San Isidro & Polpaico & 220 & 902,3 & 734,7 & 167,7 \\
\hline San Isidro & Polpaico & 220 & 902,3 & 734,7 & 167,7 \\
\hline San Isidro & Polpaico & 220 & 902,3 & 734,7 & 167,7 \\
\hline Lampa & Cerro Navia & 220 & 596,0 & $-902,0$ & $1.498,0$ \\
\hline Temuco & Puerto Montt & 220 & $4.693,0$ & 142,0 & $4.551,0$ \\
\hline Cerro Navia & Chena & 220 & 305,0 & $-199,0$ & 504,0 \\
\hline Cerro Navia & Chena & 220 & 305,0 & $-199,0$ & 504,0 \\
\hline Carera Pinto & Cardones & 220 & 1.355 .0 & $-1.536,0$ & $2.891,0$ \\
\hline Polpaico & Jahuel & 220 & $2.227,0$ & 233,0 & $1.994,0$ \\
\hline Polpaico & Jahuel & 220 & $2.227,0$ & 233,0 & $1.994,0$ \\
\hline Polpaico & Lampa & 220 & 714,0 & 152,0 & 562,0 \\
\hline Temuco & Valdivia & 220 & $2.030,0$ & $-177,0$ & $2.207,0$ \\
\hline Victoria & Temuco & 220 & 909,5 & $-861,0$ & $1.770,5$ \\
\hline Pan de Azúcar & Los Vilos & 220 & $2.923,0$ & $1.360,0$ & $1.563,0$ \\
\hline Pan de Azúcar & Los Vilos & 220 & $2.923,0$ & $1.360,0$ & $1.563,0$ \\
\hline Los Vilos & San Isidro & 220 & $1.405,0$ & $-1.214,0$ & $2.619,0$ \\
\hline Los Vilos & San Isidro & 220 & $1.405,0$ & $-1.214,0$ & $2.619,0$ \\
\hline Barros Blancos & Valdivia & 220 & $1.453,0$ & $-527,0$ & $1.980,0$ \\
\hline Jahuel & Jahuel & $500 / 220$ & $2.872,0$ & $-50,0$ & $2.922,0$ \\
\hline Jahuel & Jahuel & $500 / 220$ & $2.872,0$ & $-50,0$ & $2.922,0$ \\
\hline Ancoa & Ancoa & $500 / 200$ & $2.598,0$ & $-49,0$ & $2.647,0$ \\
\hline Ancoa & Ancoa & $500 / 200$ & $2.598,0$ & $-49,0$ & $2.647,0$ \\
\hline Total & & & $83.758,0$ & $4.808,0$ & $78.950,0$ \\
\hline
\end{tabular}

Fuente: CDEC-SIC (2003). 
TARIFICACión dE la Transmisión Eléctrica 